\title{
Deployment of an IoT Solution for Early Behavior Change Detection
}

\author{
Hamdi Aloulou ${ }^{1,2(\bowtie)}$, Mounir Mokhtari ${ }^{3}$, and Bessam Abdulrazak ${ }^{4}$ \\ 1 Centre de Recherche en Numérique de Sfax, Sfax, Tunisia \\ hamdi.aloulou@isima.u-monastir.tn \\ 2 Université de Monastir, Institut Supérieur d'Informatique de Mahdia, \\ Mahdia, Tunisia \\ ${ }^{3}$ Institut Mines Télécom, Paris, France \\ ${ }^{4}$ Université de Sherbrooke, Sherbrooke, Canada
}

\begin{abstract}
Today, numerous factors are causing a demographic change in many countries in the world. This change is producing a nearly balanced society share between the young and aging population. The noticeable increasing aging population is causing different economical, logistical and societal problems. In fact, aging is associated with chronic diseases in addition to physical, psychological, cognitive and societal changes. These changes are considered as indicators of aging peoples' frailty. It is therefore important to early detected these changes to prevent isolation, sedentary lifestyle, and even diseases in order to delay the frailty period. This paper presents an experiment deployment of an Internet of Thing solution for the continuous monitoring and detection of elderly people's behavior changes. The objective is to help geriatricians detect sedentary lifestyle and health-related problems at an early stage.
\end{abstract}

Keywords: Behavior change $\cdot$ Internet of Things $\cdot$ Frailty

\section{Introduction}

Aging is often related to significant changes in physical activities, mobility, nutrition, social life and cognitive status. These changes considerably affect elderly people quality of life. According to the World Health Organization (WHO) [1], the biggest health risk for seniors is the adoption of a sedentary lifestyle that causes isolation, depression and many other diseases such as cardiovascular disease, obesity, high blood pressure, etc.

In this paper, we present our experience in deploying an Internet of Things (IoT) solution for the continuous monitoring and detection of elderly people's behavior changes. The objective is to help geriatricians detect sedentary lifestyle and health related problems at early stage, without the need to perform classical psycho-geriatric tests that have many limitations like assessment inaccuracies

Supported by H2020 European Project City4Age.

(C) The Author(s) 2019

J. Pagán et al. (Eds.): ICOST 2019, LNCS 11862, pp. 27-35, 2019.

https://doi.org/10.1007/978-3-030-32785-9_3 
and the difficulty for elderly people to recall past events. The work was performed as part of the European project City4Age based on 6 pilot sites: Athens, Birmingham, Lecce, Madrid, Montpellier and Singapore. In this paper, we will focus and detail the deployment performed in the pilot site of Montpellier.

\section{Literature Review and Related Work}

Early detection of ageing people behavior change can improve medical assessments and enable proactive intervention. In fact, aging-related health problems generate long-term behavior changes, such as possible instabilities, variations, impairments, declines, increases or improvements [2]. Nowadays, geriatricians use psycho-geriatric scales and questionnaires to analyze behavior and investigate possible changes $[3,4]$. These psycho-geriatric approaches are insufficient to monitor patients on a daily basis [5]. Thus, geriatricians need technological services to acquire new objective observations that complete their medical observations. Monitoring technologies can help follow-up elderly people at home and in city, in order to early detect possible health changes [6].

Contrary to existing technological solutions that target Short-term Health Change Detection [7], retrospectively investigate possible changes after change occurrence [8] and use intrusive technologies to capture video sequences, collect daily questionnaire-based information and record physical data using body sensors [9], our proposed approach analyzes overall behavior over long periods, in order to detect long-term changes in health status. These long-term changes require weeks and months to emerge, and are difficult to detect due to normal continuous variation in human behavior [10]. In addition, it continuously analyzes monitoring data on a daily basis, in order to early detect possible changes. This proactive change detection provides opportunity for daily assessment and subsequent intervention. Finally, it uses unobtrusive monitoring technologies that are embedded in our real environment or in objects of daily living, do not interfere with natural behavior of elderly people and do not change their daily habits.

\section{Montpellier Pilot Setup}

Montpellier pilot site goal is to quickly and unobtrusively detect possible aging people's behavior changes. Detected behavior changes are afterwards analyzed and confirmed by collaborating geriatricians to provide adequate intervention.

The pilot site is Coordinated by the French National Center for Scientific Research (CRNS) and is collaborating with local authorities such as Montpellier Metropolis, the ETAPE (health autonomy pole) association and healthcare professionals from Beausoleil Clinic and Saint Vincent De Paul nursing home.

The proposed solution deployed in the pilot site of Montpellier consists of a set of sensors deployed in the participants' homes and in the city as shown in Fig. 1. For indoor monitoring, the pilot uses motion sensors, contact sensors 
and bed sensors. Outdoor monitoring is based on Smartphones carried by participants and beacons deployed in places of interest of participants in the city. These sensors allow to collect raw data and objective information in real time, which are processed to detect behavior changes.

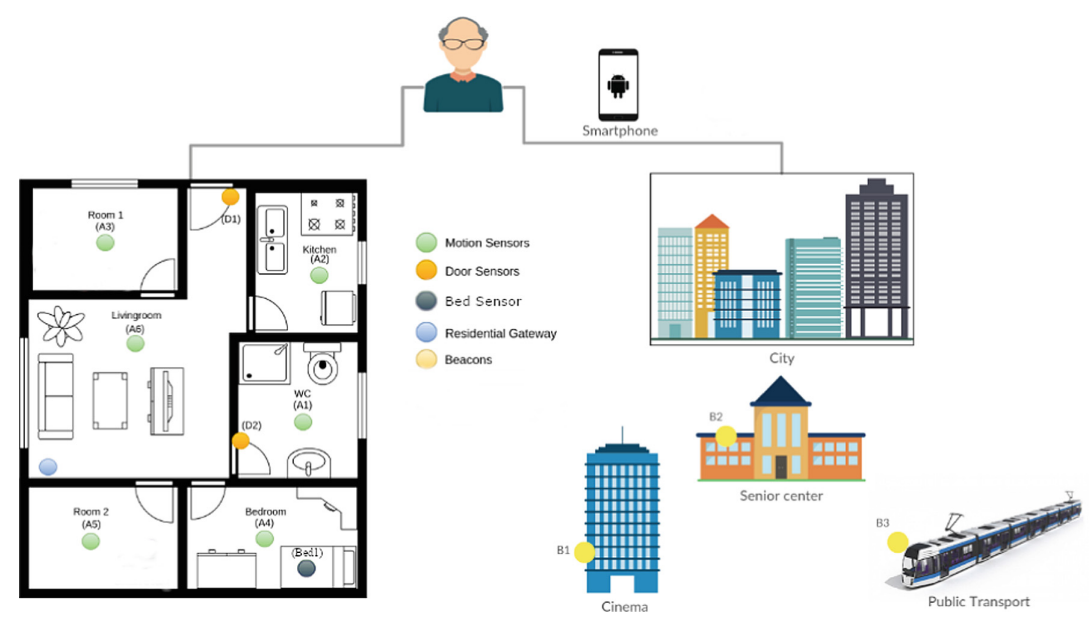

Fig. 1. Montpelier pilot site global setup

\section{Recruitment and Engagement}

Each of the six pilot sites followed a specific ethical approval process, related to specific countries' regulation, in order to recruit participants. For Montpellier pilot site, the ethical process consists in sending the specifications of the deployment to CNRS's Data Protection Correspondent (Correspondants Informatique et Libertés: CIL). In parallel, an application was also submitted to the Institutional review board (Comités de Protection des Personnes: CPP).

After obtaining the ethical approval, we started the recruitment process. We have approached around 40 potential participants with the help of ETAPE association and Saint Vincent De Paul nursing home. In fact, we have presented the solution in several local events to promote the project and identify interested people to be included in the study. As shown in Fig. 2, briefing and presentation sessions were organized in the Montpellier pilot site to better promote experimentation objectives, quickly launch recruitment process and keep recruited participants involved in the experimentation. These events allowed to have in depth discussions with interested aging people who accepted to visit a demonstration house, see a live demonstration of the system and have their feedback. The demonstration house highlights the unobtrusiveness of the technological solution proposed for the potential participants. Interested people also observe sensor events in real-time and examples of real data over weeks and months indicating significant changes in health status. Potential participants asked for 
information about employed technological solutions, real benefits of adopting them at home and possible risks.

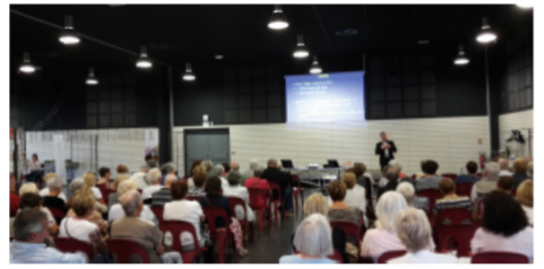

Local Medical Event "Pass'Sport Santé Séniors"

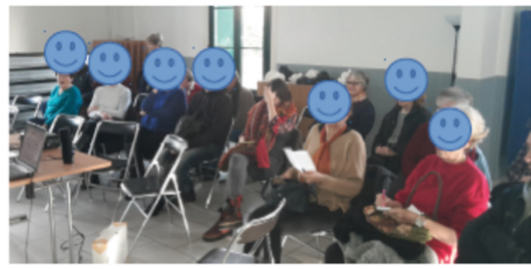

Local Medical Event "France Parkinson"

Fig. 2. Briefing and involvement sessions

19 participants accepted and have been equipped with the City4Age solution. An initial interview with included participants allowed to collect some indications on their social and health profiles. Participants have diverse medical and social profiles such as educational level, dependence level, habits and health status. Table 1 presents regular habits and health info of some participants.

Table 1. Examples of Montpelier pilot site participants habits and health status

\begin{tabular}{l|l|l}
\hline Patient & Regular habits & Health info \\
\hline 98 & $\begin{array}{l}\text { Wakes up at } 8 \mathrm{~h} . \text { Home aid } 4 \text { times per day. Stays } \\
\text { most often at home. Sometimes goes out with } \\
\text { daughter or caregiver }\end{array}$ & $\begin{array}{l}\text { Alzheimer } \\
\text { Diabetes } \\
\text { Vision and audition } \\
\text { problems }\end{array}$ \\
\hline 101 & $\begin{array}{l}\text { Wakes up at } 7 \mathrm{~h} 30-8 \mathrm{~h} \text {. Home aid visits } 3 \text { times per } \\
\text { day (morning, midday and evening). Niece and } \\
\text { neighbor visits during the day. Sleeps earlier than } \\
\text { before (at } 20 \mathrm{~h} \text {, and before at } 22 \mathrm{~h} \text { ) }\end{array}$ & $\begin{array}{l}\text { Alzheimer } \\
\text { Some falls and } \\
\text { hospitalizations }\end{array}$ \\
\hline 102 & $\begin{array}{l}\text { Wakes up at } 6 \mathrm{~h}-7 \mathrm{~h} \text {. Home aid visits each day in the } \\
\text { morning. Lives alone. Daughter house is nearby. } \\
\text { Monthly visits to and from daughter }\end{array}$ & $\begin{array}{l}\text { Heart problems } \\
\text { Urinary infection }\end{array}$ \\
\hline
\end{tabular}

\section{Technologies and Data Collection}

The system proposed in Montpellier pilot site uses indoor and outdoor technologies to monitor daily living activities of participants. For indoor monitoring, the pilot is proposing a set of sensors (motion sensors, contact sensors and bed sensors). These sensors operate discreetly and allow to collect raw data and objective information in real time. The objective is to be able to accurately determine the "habitual behaviours" of people by collecting data over time. Outdoor monitoring is based on beacons deployed in the participants' places of interest in the city 
(e.g. bus and metro stations, cinemas, restaurants, etc.) and smartphones with dedicated mobile application carried by the participants. Thus, it is possible to collect data on the activities of our participants' even when they are in outdoor in the city. Figure 3 showcases some deployed sensors in Montpellier pilot site.

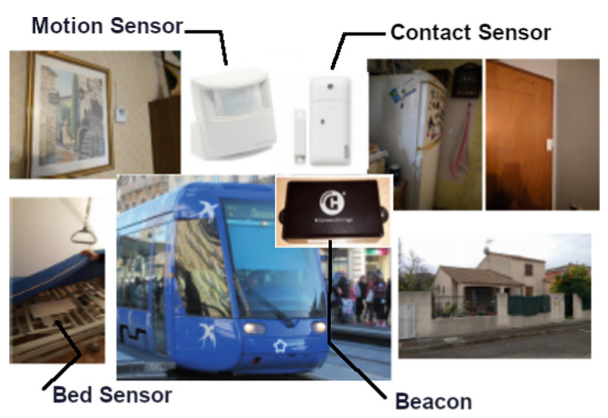

Fig. 3. Indoor and outdoor sensors deployment

All indoor and outdoor data are collected in Montpellier pilot site local server where local treatment for Low Elementary Actions (LEAs) and measures identification is performed. Low Elementary Actions are basic participants' actions which are inferred from received sensors' events (e.g. Start Moving, Stop Moving, Change Room, Visit Restaurant, etc.). Measures are quantified data extracted from LEAs (e.g time in the bedroom/day, number of toilet visits /day, number of shops visits/week, etc.). Later, these information are transferred to the City4Age repository and analytic algorithm where further treatments are performed in order to produce visualisations for the geriatrician. The complete architecture of the City4Age solution and the performed deployment and data analysis in Montpellier pilot site is presented in Fig. 4.

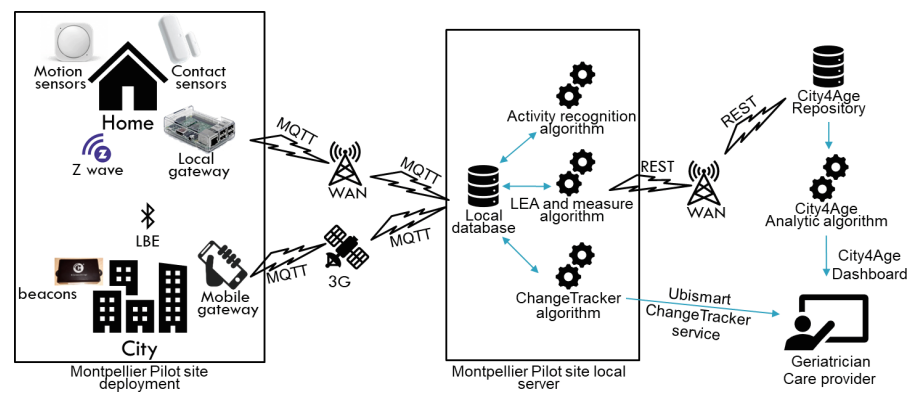

Fig. 4. Complete architecture of Montpellier pilot site's deployment 
The deployment of this system in France allowed to collect around two years of real data. We have collected 310.590 of Low Elementary Actions (LEA) and 49.659 of Measures for 19 participants.

\section{Data Interpretation}

The goal of the system proposed in Montpellier pilot site is to detect possible behavior changes that will be analysed and confirmed by collaborating geriatricians to provide adequate intervention. A behavior change tracker service [11] was developed allowing to detect changes in participants' behavior using statistical algorithms. Collected data are analyzed by this developed algorithm and presented to collaborating geriatricians from clinic Beausoleil. Figure 5 showcases some behavior changes detected by the ChangeTracker service for one of the participants in the pilot site. In Fig. 5, 3 consecutive decreases on 2017-0215, 2017-06-20 and 2017-10-25 are detected for participant 91. Participant and family doctor confirm mobility impairments and increased risk of dependence in managing activities of daily living. Professional caregiver helps with medication taking and household from 2017-05-04.

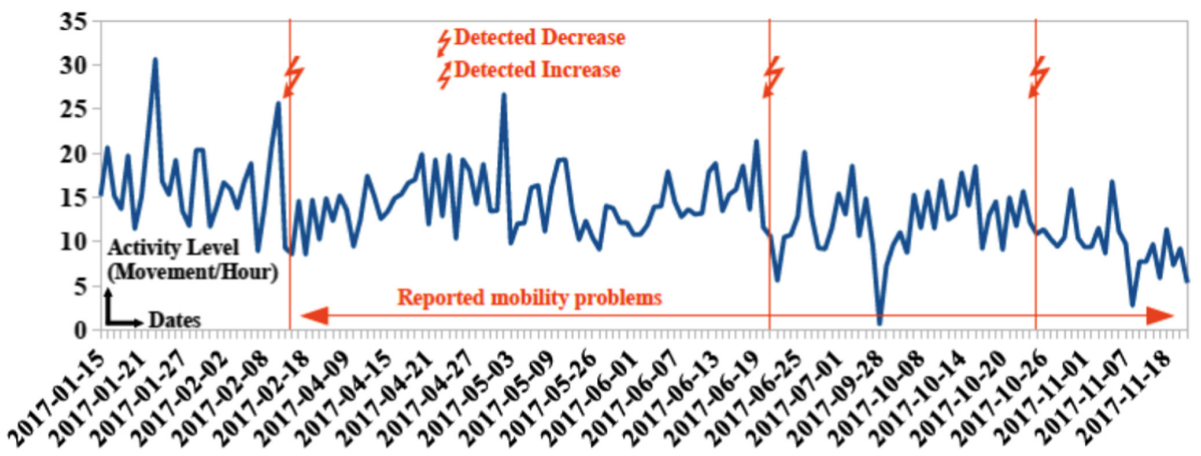

Fig. 5. Detected changes in activity level of participant 91 due to mobility impairments

\section{Intervention Process}

To perform intervention, the pilot site is providing a framework with visualizations about participants activities and statistics about their daily routines and habits. The ChangeTracker service is integrated in this framework allowing to automatically detect possible changes that can be confirmed by the caregivers and the geriatrician. The caregivers and geriatricians can navigate the data provided by these visualizations and decide on the type and form of intervention when needed. As an example, after detecting a decrease in outdoor and indoor activities for participant 96, nursing home stuff decided to initiate home assistance. 


\section{Validation}

\subsection{Detection Process Validation}

The ChangeTracker service has being validated with the help of our stakeholders. Detected changes are being correlated with medical observation and health records to validate the used algorithms and their performances.

The ChangeTracker service sends change notifications. Change notifications provide opportunity to confirm that detected changes are really permanent and investigate possible correlations with geriatric observations. Regular review meetings with elderly people, family members and family doctors allow to accurately investigate possible causes of detected changes. Review meetings investigate mutli-dimensional correlations of detected changes, such as identifying parallel decreases in activity level and time out home related to mobility impairments, and consecutive increases in sleep interruptions and toilet entries after treatment change. Figure 6 shows some results of changes detected by ChangeTracker service and their correlation with medical observations and participant feedback.

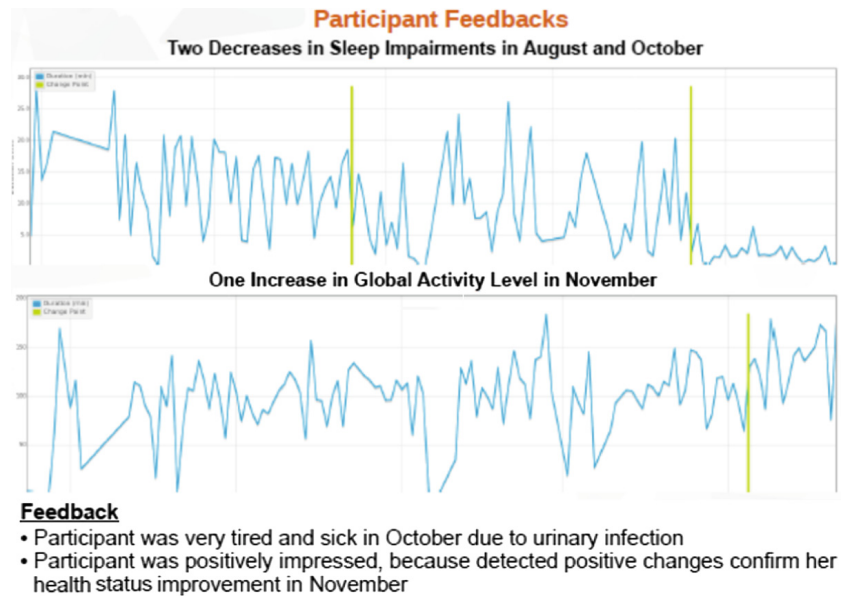

Fig. 6. Detected behavior changes by ChangeTracker and corresponding participant feedback

\subsection{Results and Performance}

In total, we have detected 340 changes for all participants with an average of 0.97 change per month. Participants show diverse changes in monitoring period that are associate with diverse medical reasons, such as physical problems $(45.59 \%$ of detected changes), health improvements (36.76\%), nutritional problems $(8.82 \%)$, personal changes $(7.35 \%)$ and social problems $(1.47 \%)$. 


\section{Conclusion}

Montpellier pilot site was a proof of concept of our IoT proposed solution for the early identification of behavior changes. In total, 340 changes have been detected for all participants. These changes have been validated and classified with the help of local geriatrician and by correlation with Medical Observations. This solution could be of great value for the geriatricians. In fact, the technological observations provided by the proposed solution enrich their medical observation for better assessments of frailty and MCI. The solution is also valuable for nursing homes. In fact, nursing homes need to maintain independent living at home and identify elderly people at risk who really require entry to nursing home.

Acknowledgment. This work was supported by the European project City4Age that received funding from the Horizon 2020 research and innovation program under grant agreement number 689731 . We would like to thank all the participants who accepted to be part of this experimentation and all our collaborating geriatricians and professional and informal caregivers.

\section{References}

1. Waxman, A.: Who global strategy on diet, physical activity and health. Food Nutr. Bull. 25(3), 292-302 (2004)

2. Cao, L.: In-depth behavior understanding and use: the behavior informatics approach. Inf. Sci. 180(17), 3067-3085 (2010)

3. Tardieu, E., et al.: External validation of the short emergency geriatric assessment (SEGA) instrument on the safes cohort. Geriatrie et psychologie neuropsychiatrie $\mathrm{du}$ vieillissement 14(1), 49-55 (2016)

4. Cockrell, J.R., Folstein, M.F.: Mini-mental state examination. In: Principles and Practice of Geriatric Psychiatry, pp. 140-141 (2002)

5. Lökk, J.: Lack of information and access to advanced treatment for Parkinson's disease patients. J. Multidisc. Healthc. 4, 433 (2011)

6. Acampora, G., Cook, D.J., Rashidi, P., Vasilakos, A.V.: A survey on ambient intelligence in healthcare. Proc. IEEE 101(12), 2470-2494 (2013)

7. Aloulou, H., et al.: Deployment of assistive living technology in a nursing home environment: methods and lessons learned. BMC Med. Inform. Decis. Making 13(1), 42 (2013)

8. Sprint, G., Cook, D.J., Schmitter-Edgecombe, M.: Unsupervised detection and analysis of changes in everyday physical activity data. J. Biomed. Inform. 63, 54-65 (2016)

9. Avvenuti, M., Baker, C., Light, J., Tulpan, D., Vecchio, A.: Non-intrusive patient monitoring of Alzheimer's disease subjects using wireless sensor networks. In: 2009 World Congress on Privacy, Security, Trust and the Management of e-Business, pp. 161-165. IEEE (2009)

10. Lally, P., Van Jaarsveld, C.H., Potts, H.W., Wardle, J.: How are habits formed: modelling habit formation in the real world. Eur. J. Soc. Psychol. 40(6), 998-1009 (2010) 
11. Kaddachi, F., Aloulou, H., Abdulrazak, B., Fraisse, P., Mokhtari, M.: Long-term behavior change detection approach through objective technological observations toward better adaptation of services for elderly people. Health Technol. 8(5), 329$340(2018)$

Open Access This chapter is licensed under the terms of the Creative Commons Attribution 4.0 International License (http://creativecommons.org/licenses/by/4.0/), which permits use, sharing, adaptation, distribution and reproduction in any medium or format, as long as you give appropriate credit to the original author(s) and the source, provide a link to the Creative Commons license and indicate if changes were made.

The images or other third party material in this chapter are included in the chapter's Creative Commons license, unless indicated otherwise in a credit line to the material. If material is not included in the chapter's Creative Commons license and your intended use is not permitted by statutory regulation or exceeds the permitted use, you will need to obtain permission directly from the copyright holder.

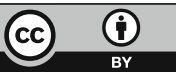

